Portland State University

PDXScholar

$11-2020$

\title{
Public Housing With Services for Older Adults With Developmental Disabilities: Differences in Efficacy Among Asian and White Communities
}

Junghee Lee

Portland State University, jungl@pdx.edu

William Donlan

Portland State University, donlan@pdx.edu

Paula C. Carder

Portland State University, carderp@pdx.edu

Leigh Grover

Portland State University, grovera@pdx.edu

Insik Hwang

Yonsei University

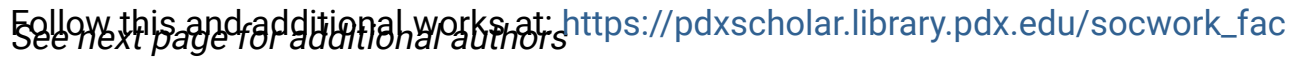

Part of the Social Welfare Commons, and the Social Work Commons

Let us know how access to this document benefits you.

\section{Citation Details}

Published as: Junghee Lee, William Donlan, Paula Carder, Leigh Grover, Insik Hwang, Younghwan Kim \& Gretchen Luhr (2020) Public Housing With Services for Older Adults With Developmental Disabilities: Differences in Efficacy Among Asian and White Communities, Journal of Aging and Environment.

This Post-Print is brought to you for free and open access. It has been accepted for inclusion in Social Work Faculty Publications and Presentations by an authorized administrator of PDXScholar. Please contact us if we can make this document more accessible: pdxscholar@pdx.edu. 


\section{Authors}

Junghee Lee, William Donlan, Paula C. Carder, Leigh Grover, Insik Hwang, Younghwan Kim, and Gretchen Luhr 
RUNNING HEAD: PUBLIC HOUSING WITH SERVICES FOR OLDER ADULTS

Public Housing with Services for Older Adults with Developmental Disabilities: Differences in Efficacy Among Asian and White Communities

Junghee Lee, William Donlan, Paula Carder, Leigh Grover, Insik Hwang, Younghwan Kim, and Gretchen Luhr

Portland State University

\section{Author Note}

Insik Hwang and Younghwan Kim are now at the School of Social Welfare, Yonsei University, Seoul, South Korea.

Gretchen Luhr is now at the Vital Research, California, USA.

We have no conflicts of interests to disclose.

Correspondence concerning this article should be addressed to Junghee Lee, $1800 \mathrm{SW} 6^{\text {th }}$ Ave, ASRC 600, School of Social Work, Portland State University. Email: jungl@pdx.edu. 


\begin{abstract}
This study examined the efficacy of a culturally-competent housing with services model for Asian-American older adults and persons with developmental disabilities separately, and with both for an interaction effect. Study participants, including 278 Whites and 75 Asian Americans, lived in 10 public housing with services properties in Portland, Oregon. All Asian-American participants (except four) were immigrants from China, Korea, and Vietnam, and were well connected through community-based health and social services that were delivered onsite and at a nearby center by staff members who were also immigrants from these three countries. AsianAmerican participants were older, very poor, with limited English proficiency, but, reported stronger family connection and satisfaction with living environment, which reflected in lower healthcare needs and utilization, and better quality of life. Participants with developmental disabilities comprised 27 individuals who reported greater healthcare needs, service utilization, and food insecurity. Asian-American participants with developmental disabilities reported stronger family connections. Regression analyses found healthcare needs was consistently the strongest predictor, followed by food insecurity and family connection, in predicting perceived quality of life. Findings offer insight into a culturally-competent housing services model where minority culture is no longer a risk factor, and LEP is no longer a cause of isolation.
\end{abstract}




\section{Public Housing with Services for Older Adults with Developmental Disabilities: Differences in Efficacy Among Asian and White Communities}

\section{Introduction}

Housing can serve as a valuable platform for accessing health services and social supports, especially for vulnerable older adults. Public housing with resident services can remove barriers and provide access for low-income older adults to much needed services. In addition, housing with services can be important for older adults with limited English language proficiency (LEP), including immigrants of Asian descent. Within the population of older adults who reside in public housing, individuals with developmental disabilities represent a small but particularly vulnerable group, especially those with LEP. While increasing attention has been given to eliciting older adults' views on successful or healthy aging, this study seeks to address the dearth of research on the health experiences and overall perceived quality of life among older adults with LEP. This study also seeks to shed some light on the experience of older Asian immigrant adults with developmental disabilities residing in public housing with resident services.

\section{Public Housing with Services}

Older adults and persons with disabilities that have low incomes and minimal resources are at a higher risk of poor health outcomes compared to more affluent counterparts (Alley et al., 2009). Barriers to health and social services, combined with poor health, can lead to housing instability and hospitalization. By coordinating access to health and social services in conjunction with affordable housing services, older adults and people with disabilities may experience better health outcomes and quality of life, while using fewer costly health services. In the U.S., over one million older adults receive housing assistance or live in a public housing 
unit funded by the Department of Housing and Urban Development (HUD) (Harvard Joint Center for Housing Studies, 2015). Among approximately 1.1 million HUD supported housing units, $31 \%$ of residents are seniors (age $62+$ ), and $30 \%$ of public housing households include family members with disabilities under age 62 (National Housing Law Project, 2017). Housing stability as a context for providing accessible health and social services to these vulnerable populations is understudied and merits greater scrutiny (Braveman \& Gottlieb, 2014; Wang et al., 2018).

\section{Diversity and Population Aging}

By 2045 the U.S. will be a 'majority-minority' country with non-Hispanic Whites making up $49.7 \%$ of the population (U.S. Census Bureau, 2018). The U.S. population is expected to rise to 438 million in 2050 , and fully $82 \%$ of the growth during this period will be due to the arrival of immigrants and their descendants. In 2050, nearly one in five Americans will be foreign-born (Batalova, 2011). The immigrant groups with the highest expected growth rates are of Hispanic and Asian/Pacific Islander descent. Among Asian immigrants, the top three countries of origin are the Philippines, India, and China followed by Vietnam and Korea.

Not only is the U.S. becoming more racially and ethnically diverse, it is also aging. The Asian-American older adult population grew by 76\% from 1990 to 2000; in comparison during this time period, the White older adult population only grew by $9.2 \%$ (U.S. Census Bureau, 2001). It is projected that the Asian-American older adult population will further grow by $246 \%$ from 2000 to 2025 (vs. 73\% among their White counterparts). Most Asian-American older adults were not born in the U.S. and immigration often occurred late in life. According to recent studies with Asian-American populations (Asian Americans Advancing Justice, 2016), about 90\% of Chinese, Vietnamese, and Korean older adults were foreign-born. About 30\% of Chinese 
and Koreans, and over $40 \%$ of Vietnamese immigrated to the U.S. after the age of 60 . Great cultural variations exist among Asian-American immigrant older adults, and due to their arrival later in life, they tend to hold on to cultures of origin more than their U.S.-born offspring (Ryan et al., 2003).

\section{Health Risk Factors among Asian-American Older Adults}

Older Asian-American immigrants come to the U.S. from many different language backgrounds. Blacher (2013) found that approximately $60 \%$ of Asian-American older adults are LEP. Not surprisingly, LEP individuals are more likely to live in poverty; about $25 \%$ of LEP individuals lived in households with an annual income below the federal poverty line, which is nearly twice as high as the rate among English-proficient persons (14\%) (Zong \& Batalova, 2015). Poverty is widespread among older Asian-American immigrants. Statistics show the poverty rate among Asian-American older adults rose 40\% between 2009 and 2014. In 2012, the poverty rate for Asian-Americans age 65+ was $13.1 \%$ (vs. 9.1\% for all older Americans) (National CAPACD, 2016).

Late-life immigrants living in the U.S. with substantial barriers to the language (Diwan, 2008; Ponce et al., 2006) and culture of mainstream society (Ra et al., 2019) are at risk. A study (Jang et al, 2016) of Korean-American older adults found LEP was a critical source of health vulnerability. More specifically, the risk of having activity limitations was 2.72 times higher, a fair or poor rating of health 2.59 times greater, and probable depression 1.73 times more likely among participants with LEP than counterparts. In a study with Chinese- and Korean-immigrant older adults, acculturation stress in the form of poor English proficiency was associated with poor health outcomes and health-related quality of life (Mui et al., 2007). Although family support is perceived as a strong positive asset (Guo et al, 2015), interestingly, living 
arrangements and frequency of contact with both kin and non-kin networks were found to only weakly correlate with depression (Guo \& Stensland, 2017). Strong correlations were found between depression and support with family (e.g., Guo et al., 2015) and connection with friends (Lee \& Kim, 2014). Higher levels of depression were observed among Asian-American older adults who experienced lower levels of community connection, limited participation in ethnic community events and activities, and less frequent interactions with ethnic community members (Jang et al., 2015).

\section{Aging and Health Among People with Developmental Disabilities}

Developmental disability is defined as a severe, long-term disability that can affect cognitive ability, physical functioning, or both (NIH, 2010), which is caused by having one or more of three diagnoses: autism spectrum disorder, intellectual disability, and other developmental delay (Zablotsky et al., 2017). Developmental disability appears before age 22 and is typically life-long. A recent study estimates that about $15 \%$ of U.S. children have one or more developmental disabilities (Boyle et al., 2011). As a result of an aging society, the number of adults with developmental disabilities aged $60+$ is projected to nearly double from 641,860 in 2000 to 1.2 million by 2030 (Tinglin, 2013).

Adults with developmental disabilities are more likely to develop chronic health conditions at younger ages than their counterparts because of biological factors related to syndromes, limited access to healthcare, and lifestyle and environmental issues associated with developmental disabilities, which may explain the higher mortality risk and reduced life expectancy (e.g., Lauer \& McCallion, 2015) among this population. The World Health Organization (2012) reports that people with disabilities have less access to healthcare services that meet their needs. Along with their greater healthcare needs, adults with developmental 
disabilities experience serious challenges in finding healthcare providers who are competent in working with this population (e.g., Heller, 2017).

Not only a lack of adequately prepared primary healthcare professionals, but also unaffordability provides an additional challenge for people with developmental disability in accessing appropriate healthcare. People with developmental disabilities are more likely to live in poverty and, as result, a large proportion of this population relies on publicly financed healthcare, which often does not adequately meet their needs (Ervin et al., 2014). Low-income people with developmental disabilities experience greater difficulties meeting their basic daily living needs, which in turn can lead to a lower perceived quality of life and increased healthcare service utilization. Adults who have developmental disabilities have been found to have significantly worse quality of life scores when compared with the general population, notably experiencing higher rates of loneliness and reporting less social connection and stress as a correlate to poor quality of life (e.g., Lin \& Huang, 2017). Older adults in general, including Asian-American immigrants with developmental disabilities, are aging at unprecedented rates and experiencing unique healthcare and service needs (e.g., Sutherland et al., 2002). However, sadly, there is a total lack of study of older Asian-American immigrants with developmental disabilities addressing their healthcare needs and utilization, and related overall perceived quality of life.

\section{Study Purpose}

Asian-American older adults, and individuals with developmental disabilities are hard-toreach populations, and public discourse about the graying U.S. population has failed to acknowledge the unique challenges facing these two groups. Older Asian-American immigrants with developmental disabilities are even harder to access and there are no studies addressing this 
population. This study aims to better understand the health experiences and overall perceived quality of life among Asian-American immigrant older adults (vs. non-Hispanic White counterparts), and among older adults with developmental disabilities ( $v s$. without disabilities) separately, and then interaction effects between these two factors. To the best of our knowledge, this is the first relatively large-scale study with these two hard-to-reach populations, especially living in public housing with services. Health is a complex and dynamic construct; this study takes account of various social determinants of health measures (Nguyen, Lee, Sorkin \& Gibbs, 2019; Sutherland et al., 2002) such as social connection, food insecurity, and satisfaction with living environment, beyond known healthcare factors such as healthcare activities, needs, and service utilization.

\section{Methods}

\section{Study Setting}

In 2014, the Oregon Health Authority received a State Innovation Models grant from the Centers for Medicare and Medicaid Services. This grant supported building the Housing with Services Project (HWS) infrastructure and activities to provide culturally specific programs for culturally diverse residents in 10 low-income apartment buildings in Portland, Oregon. Each building was designated for individuals who met the following criteria: low or very low income, age 50 or older, or qualifying disability. Residents had to be capable of living independently, although they might receive health-related services and supports from friends and family or community organizations. This study was approved by the Institutional Review Board at Portland State University. 


\section{Recruitment and Sample}

Paper surveys were delivered to the doors of all 1,401 residents of the 10 properties. The envelope included information in various languages and offering interpretation assistance. The response rate was $39 \%$, with 541 completed questionnaires, which provided the data used for this study. Participants self-identified their racial/ethnic category and subsequently three racial groups were created: non-Hispanic White $(n=331)$, Asian American $(n=95)$, and Others $(n=$ 98) (17 declined to identify their racial group). For a comparative study between Asian Americans vs. Whites, Others were excluded from data analysis. Then, to better understand healthcare experiences among near-older and older adults (HUD, 2003), participants who were younger than $50(n=72)$ were excluded from this study along with those who failed to identify their age. Final study participants included 278 non-Hispanic Whites and 75 Asian Americans that were all age 50+. Participants were also asked to indicate if a doctor or other health professional had ever told them that they had a developmental disability. Of 27 individuals with developmental disability, 23 were Whites; only four were Asian Americans.

\section{Measures}

Healthcare Conditions. Healthy activities were measured by asking if participants exercised, received a flu shot, or had a health screening in the past 30 days. If respondents participated in none of these activities, they were recorded with 0 ; if they participated in all three activities, it was identified as 3. Healthcare needs were measured by the perceived severity of mobility challenges, pain/discomfort, usual activities, self-care, and anxiety/depression. Severity was identified on a 0-2 scale with higher scores indicating more severity. Healthcare service utilization was measured by five items that asked: in the past 6 months, how many times did you go to a doctor's office, other healthcare provider, clinic, hospital emergency room, and admitted 
overnight to a hospital. If a participant had an episode, this was entered as 1 , otherwise 0 . Total scores of each study variable were included in data analysis.

Social Determinants of Health Factors. Perceived level of family and friend connections were measured by three items separately on a 6-point Likert scale: how many friend/family members study participant (a) see or hear from at least once a month, (b) feel enough at ease with that they can talk about private matters, (c) feel close enough that they could call on them for help. Satisfaction was measured by two items: one with the apartment building as a place to live; and the other with the neighborhood as a place to live. Participants also identified overall satisfaction with their living environment on a 5-point Likert scale. Food insecurity was identified by three items: in the past 30 days, (a) were you ever concerned about having enough food to eat, (b) have you ever eaten less due to not having enough money to buy food, and (c) did you ever not eat due to not being able to get out for food. If a participant had an episode, then was coded as 1 , otherwise 0 . Total scores of each study variable were entered for data analysis purpose.

Outcome Variable. The study outcome variable was a subjective measure of overall quality of life, which was identified with one item ranging from $0=$ Worst to $100=$ Best possible quality of life. This item was stated alongside a visual analogue scale for a participant to pin down their subjective level of quality of life.

\section{Data Analysis}

A series of independent samples $t$-tests was conducted to compare group mean differences between race (White vs. Asian American) and developmental disability status (having vs. not having) on study variables as well as outcome variable separately. Several 2x2 ANOVA tests were followed to explore if interaction effects occurred between two conditions. 
OLS hierarchical regression was employed to examine how well a new set of study variables predicted the outcome variable over and above previously entered set of variables.

\section{Results}

\section{Descriptive Analysis}

Participant's mean age was $68.12(S D=9.97)$. Of 354 participants, $75(21.1 \%)$ were Asian American, 27 (7.6\%) indicated having developmental disabilities, and 185 (52.1\%) were female. Asian-American participants were more likely to be female $(p<.05)$, and married/partnered $(p<.001)$. All but two Asian-American participants were foreign-born and from: China $(n=46,60 \%)$, Korea $(n=14,19 \%)$, Vietnam $(n=10,13 \%)$, Philippines $(n=2)$ and Laos $(n=1)$. All spoke Asian languages as their primary except one who was English-speaking. Out of 10 HWS properties, Asian-American participants tended to live in the same buildings. Among 46 China-born participants, 43 lived in one building; among 14 Korea-born participants, nine lived in one building and four in another; 10 Vietnam-born participants lived in four buildings (4/4/1/1 participants); two Filipinos lived in one building. Among White study participants, only $19(6.8 \%)$ were foreign born, 23 were individuals with developmental disabilities and lived wide spread in all 10 buildings. While all residents had low incomes as a condition of housing eligibility, 82 respondents stated having Supplemental Security Income (SSI) as their primary source of income. Asian-American participants were even poorer than their counterparts $(p<.001)$, and about $80 \%$ of them reported SSI as their primary source of income.

\section{Comparative Analysis}

Independent $t$-tests found (see Table 1): Asian American (vs. White) participants were significantly older $(p<.001)$ and had stronger family connection $(p<.001)$; they reported less 
healthcare needs $(p<.01)$ and less healthcare service utilization $(p<.001)$. Asian-American participants experienced stronger satisfaction with their living environment $(p=.082)$. Participants with developmental disabilities ( $v s$. without disabilities) were younger $(p<.01)$; and indicated greater healthcare needs $(p<.05)$ and healthcare utilization $(p<.01)$, lower perceived quality of life $(p<.01)$, and higher food insecurity $(p=.076)$.

Several 2x2 ANOVA tests assessed interaction effects between race (White $v s$. Asian American) and developmental disability status (having $v s$. not-having) on each study variable separately. Because of the largely unequal sample sizes in each group, the homogeneity of variance assumption was carefully assessed, and no concerns were found. A significant interaction effect was found on family connection $\left(F(1,344)=4.08, p<.05\right.$, partial $\left.\eta^{2}=.01\right)$ alongside a significant main effect for race, $\left(F(1,344)=14.56, p<.001\right.$, partial $\left.\eta^{2}=.040\right)$, and non-significant effect for developmental disability status. Because the interaction between race and disability status was significant, the race main effect was ignored and instead the race simple main effect, that is, the differences between race with having $v s$. not-having developmental disabilities were tested separately. Asian-American participants with developmental disabilities (vs. without developmental disabilities) indicated higher family connection $(F(1,346)=19.10, p$ $<.001$ ), but interestingly no significant difference was found between Whites with $v s$. without developmental disabilities $(F(1,346)=1.52, p=.218)$.

[Insert Table 1 about here]

\section{OLS Regression Analysis}

Three sets of OLS hierarchical regression analyses were conducted and all models were significant with sufficient model description. The results of Model 1 demonstrated that healthy activities, healthcare needs and utilization accounted for a significant amount of variation in 
perceived quality of life $\left(\mathrm{R}^{2}=.28, p<.001\right)$, indicating older adults who engaged in more healthy activities $(\beta=.15, p<.01)$, experienced less healthcare needs $(\beta=-.46, p<.001)$, and utilized less healthcare $(\beta=-.13, p<.05)$, and tended to have higher scores on perceived quality of life.

A second analysis was conducted to evaluate whether a set of new variables predicted the outcome variable over and above the previous set of healthcare conditions. The four social determinants of health variables in Model 2 accounted for a significant additional proportion of the outcome variable after controlling for effects of healthcare conditions $\left(\mathrm{R}^{2}\right.$ change $=.09, p$ $<.001)$. Results suggested participants who had more connection with family $(\beta=.13, p<.01)$, with friends $(\beta=.10, p<.10)$, and experienced less food insecurity $(\beta=-.22, p<.001)$, reported higher quality of life. The three healthcare condition variables remained significant in Model 2.

In Model 3 entering a set of new variables including Asian American (vs. White) and developmental disability status ( $v s$. without disability) did not add statistically significant model description to the outcome variable $\left(\mathrm{R}^{2}\right.$ change $\left.=.01, p=.072\right)$, controlling for the two previous set of variables. As expected with older adults, experiencing more healthcare needs was consistently the strongest predictor in all three models $(\beta=-.46,-.39,-.40$ respectfully), and food insecurity was the second strongest ( $\beta=-.22$ and -.21 in Model 2 and 3 respectfully) followed by family connection ( $\beta=.13$ and .14 in Model 2 and 3 respectfully) in predicting quality of life.

[Insert Table 2 about here]

\section{Discussion}

Study findings highlight the necessity to develop a community-based and comprehensive service delivery model that improves healthcare conditions and increases food security for highly vulnerable and at-risk populations to encourage healthy independent living. Because of 
increasing life expectancy, the population of older adults living with functional impairment and developmental disability is rapidly growing (Heller, 2017; Thompson et al., 2012). Older adults with developmental disabilities experience greater healthcare needs, which often results in increased healthcare service utilization (Sutherland et al., 2002; Tinglin, 2013). As seen in our study, living with developmental disability exacerbates health risks. Older adults with developmental disabilities are less likely to be able to go out for food, and more likely to eat less than they feel they should have. While food insecurity among U.S. households remains a significant health and social problem, there are very limited studies on this topic with older adults (Brucker \& Coleman-Jensen, 2017; Goldberg \& Mawn, 2014). Furthermore, the authors were unable to find any studies that addresses food insecurity among older adults with developmental disability living in public housing. Study findings highlight the necessity for a greater understanding of the complex needs of diverse older adults with developmental disabilities to encourage healthy independent living.

The vast majority of Asian-American study participants were very poor, older, and foreign-born immigrants with LEP; all of which were identified as risk factors for health and quality of life in previous studies (e.g., Diwan, 2008; Kim et al., 2015; Jang et al., 2016). As noted in our literature review (Jang et al., 2015; Mui et al., 2007; Ryan et al., 2003), isolation manifested in loneliness, especially among very poor, older, immigrant adults, is a serious health risk that contributes to poor mental and physical health outcomes, which in turn contribute to a lessened perceived quality of life. Study findings suggest that a higher level of family connection may partially explain why the presence of greater health risks did not lower health outcomes and reduce perceived quality of life among immigrant Asian-American older adult participants. In our study, immigrant Asian-American older adults with developmental 
disabilities reported stronger family connection than their counterparts, which was not observed among White participants. However, due to their very small sample size, this finding should not be generalized, but it provides possible further insight into the greater importance of family connection among immigrant Asian-American older adults. In addition, Asian-American participants in general were living in an environment where they were able to maintain strong relationships with family, people from their home countries, communicate in languages from their country of origin, and share and celebrate their culture.

As noted in our literature review (Nguyen, Lee, Sorkin \& Gibbs, 2019; Sutherland et al., 2002), among those Asian-American older adults who immigrated to the U.S. in later life, changes in family structure and living arrangements were identified as contributing to isolation. Those who experience LEP and are unfamiliar with public transportation systems, as result tend to spend more of their days at home "living alone" or "having no one to talk to," which can exacerbate feelings of isolation, loneliness and abandonment. Our findings resonate with these previous studies, in that our study participants from China, Korea and Vietnam were well connected through community-based health and social services, which were delivered by staff members from a nearby center who were also immigrants from these three countries. Center staff were stationed in one of the public housing buildings where they were able to maintain continuous contacts and provide services. Being connected among Asian-Americans through the center staff and services appeared to have a strong influence on participants' daily lives. While this may be perceived as "cultural segregation," this can also be understood as a "culturally connected" living environment where minority culture is no longer a risk factor, and LEP is no longer a cause of isolation. In this type of culturally connected environment, some typical risk factors for reducing quality of life for immigrant older adults appear to be neutralized or 
overcome by a sense of community connection. A culturally responsive HWS model can more effectively reduce various health risk factors (healthcare needs and utilization, and food insecurity), and promote protective factors (healthy activities and social support) among poor older adults especially for immigrants with LEP. In this HWS model, perhaps one of the most important features lies in how it addresses the risks posed by isolation and loneliness through connecting residents to build a community and sense of belonging (Jang et al., 2015; Nguyen, Lee, Sorkin \& Gibbs, 2019).

\section{Implications and Conclusion}

This is a relatively large-scale study with very hard-to-reach populations. However, as the sample size of immigrant Asian-American older adults with developmental disabilities was of insufficient to achieve statistical significance, future studies with this population should consider the addition of qualitative methods such as focus groups to (a) overcome the inevitable limitations posed by small sample sizes, and (b) provide greater insight into their experiences. Study findings $d o$ offer unique insight into a relatively new culturally responsive HWS model in which the cycle of housing instability and isolation can be intentionally interrupted. This study used one item for measuring perceived quality of life, which was very simple and easily understood among our study participants. In consideration of the complex nature of this construct, it is suggested for future study to consider the utilization of a more multidimensional measure (e.g., health-related quality of life, HRQOL; Thompson et al., 2012). In addition, as the population of the U.S. continues to age and diversify, and the number of people living with functional limitations continues to grow, age relevant and culturally sensitive measures of quality of life are recommended for future study. Furthermore, developmental disability status was measured by one self-reported item. To improve the validity of this measure, it is strongly 
recommended that researchers seek to identify more specific symptoms in order to help participants better understand the definition and identify relevant symptoms.

\section{References}

Alley, D.E., Soldo, B.J., Paga'n, J.A., McCabe, J., deBlois, M., Field, S.H., Asch, D.A., \& Cannuscio, C. (2009). Material resources and population health: Disadvantages in health care, housing, and food among adults over 50 years of age. American Journal of Public Health, 99(S3), S693-S701.

Asian Americans Advancing Justice (2016). A community of contrasts: Asian Americans 50 and older. Retrieved from https://www.aarp.org/content/dam/aarp/home-and-family/asiancommunity/2016/04/2016-aaaj-report-aarp.pdf

Batalova, J. (2011). Asian immigrants in the United States. Migration Policy Institute. Retrieved from https://www.migrationpolicy.org/article/asian-immigrants-united-states-0

Blacher, K. (2013). Asian Americans and Pacific Islanders in the United States aged 65 years and older: Population, nativity, and language. Data Brief, 1(3), pp. 1-7.

Boyle, C.A., Boulet, S., Schieve, L.A., Cohen, R.A., Blumberg, S.J., Yeargin-Allsopp, M., et al. Trends in the prevalence of developmental disabilities in U.S. children, 1997-2008. Pediatrics 127(6),1034-42. 2011.

Braveman, P., \& Gottlieb, L. (2014). The social determinants of health: It's time to consider the causes of the causes. Public Health Reports, 129(Suppl 2), 19-31.

Brucker, D.L. \& Coleman-Jensen, A. (2017). Food insecurity across the adult lifespan for persons with disabilities. Journal of Disability Policy Studies, 28(2), 109-118. doi: $10.1177 / 1044207317710701$ 
Diwan S. (2008). Limited English proficiency, social network characteristics, and depressive symptoms among older immigrants. Journal of Gerontology, 63(3), S184-S191. https://doi.org/10.1093/geronb/63.3.S184

Ervin, D.A., Hennen, B., Merrick, J., \& Morad, M. (2014). Healthcare for persons with intellectual and developmental disability in the community. Frontiers in Public Health, 2(83). doi:10.3389/fpubh.2014.00083

Goldberg, S., \& Mawn, B. (2014). Predictors of food insecurity among older adults in the United States. Public Health Nursing, 32(5), 397-407. doi: 10.1111/phn.12173.

Guo, M., Li, S., Liu, J. \& Sun, F. (2015). Family relations, social connections, and mental health among Latino and Asian older adults. Research on Aging, 37, 123-147.

Guo, M., \& Stensland, M. (2017). A systematic review of correlates of depression among older Chinese and Korean immigrants in the United States: What we know and don't know. Aging \& Mental Health. doi:10.1080/13607863.2017.1383971

Heller, T. (2017). Service and support needs of adults gaining with intellectual/developmental disabilities: Testimony to the U,S, Senate Committee on Aging. Retrieved from https://aadmd.org/sites/default/files/Bridging\%20Aging\%20and\%20Intellectual $\% 20 \% 28$ $\underline{2 \% 29 t e s t i m o n y \% 20 f i n a l 103017 . p d f}$

Jang, Y., Yoon, H., Park, N., \& Chiriboga, D. (2016). Health vulnerability of immigrants with limited English proficiency: A study of older Korean Americans. Journal of the American Geriatrics Society, 64(7), 1498-1502.

Jang, Y., Park, N.S., Chiriboga, D.A., Yoon, H., An, S., \& Kim, M.T. (2015). Social capital in ethnic communities and mental health: A study of older Korean immigrants. Journal of Cross-Cultural Gerontology, 30, 131-141. doi:10.1007/s10823-015-9258-9. 
Kim, H.J., Park, E., Storr, C.L., Tran, K., \& Juon, H.S. (2015). Depression among AsianAmerican adults in the community: Systematic review and meta-analysis. PLOS one, 10(6), e0127760. doi:10.1371/journal.pone.0127760

Lauer, E., \& McCallion, P. (2015). Mortality of people with intellectual and developmental disabilities from Select US State Disability Service Systems and Medical Claims Data. Journal of Applied Research in Intellectual Disabilities, 28(5), 394-405.

Lee, S. H., \& Kim, Y.B. (2014). Which type of social activities decrease depression in the elderly? An analysis of a population-based study in South Korea. Iranian Journal of Public Health, 43(7), 903-912.

Nguyen, H., Lee, J., Sorkin, D. H., Gibbs, L. (2019). Living happily despite having an illness: Perceptions of healthy aging among Korean American, Vietnamese American, and Latino older adults. Applied Nursing Research, 48, 30-36.

Lin, L.Y., \& Huang, P.C. (2017). Quality of life and its related factors for adults with autism spectrum disorder. Disability and Rehabilitation, 41(8), 896-903. https://doi.org/10.1080/09638288.2017.1414887

Mui, A.C., Kang, S., Kang, D., \& Domanski, M.D. (2007). English language proficiency and health-related quality of life among Chines and Korea immigrant elders. Health \& Social Work, 32(2), 119-127.

National CAPACD, 2016). Findings on financial security for AAPI seniors and their families: A landscape assessment \& community survey results. https://www.aarp.org/content/dam/aarp/home-and-family/asiancommunity/2016/09/capacd-report-aarp-2016.pdf 
National Housing Law Project. (2017, October 21). Key components of public housing. Retrieved from https://www.nhlp.org/wp-content/uploads/2018/01/NHLP-Public-Housingoutline.pdf

Ponce, N. A., Hays, R. D., Cunningham, W. E. (2006). Linguistic disparities in health care access and health status among older adults. Journal of General Internal Medicine, 21, 786-791.

Ra, C, Huh, J., Finch, B., \& Cho, Y. (2019). The impact of perceived discrimination on depressive symptoms and the role of differentiated social support among immigrant populations in South Korea. International Journal for Equity in Health, 18(1):7. doi: $10.1186 / \mathrm{s} 12939-019-0910-9$

Ryan, A., Mui, A., \& Cross, P. (2003). Asian American elders in New York City: A study to assess health, social needs, quality of life and quality of care. New York: Asian American Federation of New York.

Sutherland, G., Couch, M.A., \& Iacono, T. (2002). Health issues for adults with developmental disability. Research in Developmental Disabilities, 23(6), 422-445.

Tinglin, C.C. (2013). Adults with intellectual and developmental disabilities: A unique population. Today's Geriatric Medicine, 6(3), 22.

Thompson, W., Zack, M., Krahn, G., Andresen, E., \& Barlie, J. (2012). Health-related quality of life among older adults with and without functional limitations. American Journal of Public Health, 102(3), 496-502.

United State Census Bureau. (2018). Older people projected to outnumber children for first time in U.S. history. https://www.census.gov/newsroom/press-releases/2018/cb18-41population-projections.html. 
U.S. Department of Housing and Urban Development. (2003, June). Public housing occupancy guidebook. Retrieved from https://www.hud.gov/sites/documents/DOC_10760.PDF

Wang, J., Huang, B., Zhang, T., Wong, H., Huang, Y. (2018). Impact of housing and community conditions on multidimensional health among middle- and low-income groups in Hong Kong. International Journal of Environmental Research and Public Health, 15(6), 1132. doi: $10.3390 /$ ijerph15061132

Zablotsky, B., Black, L.I., \& Blumberg, S.J. (2017). Estimated prevalence of children with diagnosed developmental disabilities in the United State, 2014-2016. NCH Data Brief, $291,1-7$

Zong, J., \& Batalova, J. (July 8, 2015). The limited English proficient population in the United States. https://www.migrationpolicy.org/article/limited-english-proficient-populationunited-states. 
Table 1.

Statistical Comparisons with Race and Developmental Disability Status on Study Variables

\begin{tabular}{|c|c|c|c|c|c|}
\hline & $\begin{array}{c}\text { White } \\
(n=278)\end{array}$ & $\begin{array}{c}\mathrm{AA} \\
(n=75)\end{array}$ & $\begin{array}{c}\text { NDD } \\
(n=325)\end{array}$ & $\begin{array}{c}\text { PDD } \\
(n=27)\end{array}$ & Stat. Comparison \\
\hline \multicolumn{6}{|l|}{ Demographic Variables } \\
\hline Male $(n, \%)$ & $124(47.5)$ & $26(36.1)$ & $167(45.5)$ & $17(48.6)$ & $n / a$ \\
\hline Female $(n, \%)$ & $135(52.5)$ & $46(63.9)$ & $200(54.5)$ & $18(51.4)$ & \\
\hline Age $($ range $=50-94)$ & $65.88(8.96)$ & $76.05(9.20)$ & $68.48(9.83)$ & $63.26(9.67)$ & $\mathrm{AA}>\mathrm{W} ; \mathrm{NDD}>\mathrm{PDD}$ \\
\hline \multicolumn{6}{|l|}{ Healthcare Conditions } \\
\hline $\begin{array}{l}\text { Health activities } \\
\text { (range=1-3) }\end{array}$ & $2.28(0.83)$ & $2.37(0.71)$ & $2.31(0.82)$ & $2.15(0.73)$ & $n s$ \\
\hline $\begin{array}{l}\text { Healthcare needs } \\
\text { (range }=0-8)\end{array}$ & $2.55(1.76)$ & $1.88(1.73)$ & $2.36(1.75)$ & $3.07(1.90)$ & $\mathrm{W}>\mathrm{AA} ; \mathrm{PDD}>\mathrm{NDD}$ \\
\hline $\begin{array}{l}\text { Healthcare utilization } \\
\text { (range }=0-12)\end{array}$ & $3.20(2.43)$ & $2.18(1.46)$ & $2.87(2.22)$ & $4.41(2.78)$ & $\mathrm{W}>\mathrm{AA} ; \mathrm{PDD}>\mathrm{NDD}$ \\
\hline \multicolumn{6}{|c|}{ Social Determinants of Health Factors } \\
\hline $\begin{array}{l}\text { Friend connection } \\
\text { (range }=0-15)\end{array}$ & $7.20(3.61)$ & $7.65(3.52)$ & $7.27(3.59)$ & $7.22(3.76)$ & Ns \\
\hline $\begin{array}{l}\text { Family connection } \\
\text { (range }=0-15)\end{array}$ & $4.70(3.97)$ & $6.83(3.65)$ & $5.19(4.00)$ & $4.59(4.16)$ & $\begin{array}{c}\text { AA_PDD }>\text { All other- } \\
\text { groups }\end{array}$ \\
\hline $\begin{array}{l}\text { Satisfaction } \\
\text { (range=1-10) }\end{array}$ & $7.74(2.13)$ & $8.13(1.59)$ & $7.87(2.05)$ & $7.33(1.69)$ & $N S$ \\
\hline $\begin{array}{l}\text { Food insecurity } \\
\text { (range }=0-3 \text { ) }\end{array}$ & $0.60(1.02)$ & $0.68(0.94)$ & $0.58(0.98)$ & $1.04(1.20)$ & $\mathrm{PDD}>\mathrm{NDD}$ \\
\hline \multicolumn{6}{|l|}{ Outcome Variable } \\
\hline $\begin{array}{l}\text { Quality of Life } \\
\text { (range=0-100) }\end{array}$ & $63.53(22.79)$ & $67.04(19.78)$ & $65.02(22.26)$ & $49.70(23.79)$ & NDD $>$ PDD \\
\hline
\end{tabular}

Note. Independent samples $t$-test compared race and development disability status separately. ANOVA test found an interaction effect on Family connection. NDD: Persons without developmental disabilities. PDD: Persons with developmental disabilities. AA: Asian American. 
Table 2.

Regressing Quality of Life by Study Variables

\begin{tabular}{|c|c|c|c|}
\hline \multirow[b]{3}{*}{ Healthcare Conditions } & \multicolumn{3}{|c|}{ Standardized Coefficients } \\
\hline & Model 1 & Model 2 & Model 3 \\
\hline & \\
\hline Health activities & $0.15 * *$ & $0.11^{*}$ & $0.11^{*}$ \\
\hline Healthcare needs & $-0.46^{* * *}$ & $-0.39 * * *$ & $-0.40 * * *$ \\
\hline Healthcare utilization & $-0.13^{*}$ & $-0.11 *$ & $-0.11 *$ \\
\hline \multicolumn{4}{|l|}{ Social Determinants of Health Factors } \\
\hline Friend connection & & $0.10^{+}$ & $0.10^{*}$ \\
\hline Family connection & & $0.13 *$ & $0.14 * *$ \\
\hline Satisfaction with living environment & & 0.06 & 0.06 \\
\hline Food insecurity & & $-0.22 * * *$ & $-0.21 * * *$ \\
\hline \multicolumn{4}{|l|}{ Demographic Variables } \\
\hline Asian American (vs. White) & & & -0.07 \\
\hline $\begin{array}{l}\text { Having developmental disability } \\
\text { (vs. Not having disability) }\end{array}$ & & & $-0.09^{+}$ \\
\hline \multirow[t]{3}{*}{ Model Summary } & $F(3,296)=38.88^{* * *}$ & $F(7,292)=25.29 * * *$ & $F(9,290)=20.49^{* * *}$ \\
\hline & $\mathrm{R}^{2}=.283$ & $\mathrm{R}^{2}=.377$ & $\mathrm{R}^{2}=.389$ \\
\hline & \multicolumn{3}{|c|}{$\Delta \mathrm{R}^{2}=.095^{* * *} \quad \Delta \mathrm{R}^{2}$} \\
\hline
\end{tabular}

Note. ${ }^{* *}: p<.001 ; * *: p<.01 ; *: p<.05{ }^{*}{ }^{*}: p<.10$ 